\title{
DE LA LITTÉRATURE
}

\section{À LA PHILOSOPHIE:}

\section{DELEUZE INTERCESSEUR}

L'être qui était rené en moi quand avec un tel frémissement de bonheur $j$ 'avais entendu le bruit commun à la fois à la cuiller [...] et au marteau [...], à l'inégalité pour les pas des pavés de la cour de Guermantes [...], cet être-là ne se nourrit que de l'essence des choses, en elles seulement il trouve sa subsistance, ses délices. Il se languit dans l'observation du présent où les sens ne peuvent la lui apporter, dans la considération d'un passé que l'intelligence lui dessèche, dans l'attente d'un avenir que la volonté construit avec des fragments du présent et du passé auxquels elle retire encore de leur réalité ne conservant que ce qui convient à la fin utilitaire, étroitement humaine qu'elle leur assigne. Mais qu'un bruit, qu'une odeur, déjà entendu et respirée jadis le soient de nouveau, à la fois dans le présent et dans le passé, réels sans être actuels, idéaux sans être abstraits, aussitôt [...] notre vrai moi qui, parfois, depuis longtemps, semblait mort, mais ne l'était pas autrement, s'éveille, s'anime en recevant la céleste nourriture qui lui est apportée.

Marcel Proust - Le Temps retrouvé II.

Nous appréhendons une qualité sensible comme signe; nous sentons un impératif qui nous force à en chercher le sens. Alors il arrive que la mémoire involontaire, 
directement sollicitée par le signe, nous livre ce sens (Venise pour les pavés).

Gilles Deleuze - Proust et les Signes.

Ces sensations liées à la venue précoce de la nuit sur la ville hivernale, ou bien, peu après la rentrée des classes, vers la fin de l'automne, quand les devantures vétustes des boulangeries ou épiceries de quartier s'éclairent déjà tôt, tandis qu'il fait encore assez doux et qu'une pluie très fine parsème d'éclats brillants les rues au pavage inégal [...] ces sensations très vives (bien que paisibles) de repos vespéral, de lampes accueillantes, de rumeur diffuse et comme lointaine, de soupe aux légumes, d'abat-jour au papier roussi, j'ai signalé souvent que je voyais là une des raisons - sinon la principale - qui m'avaient poussé vers le roman. Je comprends très bien ce que signifie: se mettre à écrire à cause de la couleur jaune aperçue sur un vieux mur.

Alain Robbe-Grillet - Le Miroir qui revient.

Dans un livre récent sur les textes communs du philosophe discrètement brillant Gilles Deleuze et de son ami le psychanalyste Felix Guattari, Charles J. Stivale se propose d'examiner leurs écrits à partir des "intersections" entre les concepts produits par les deux auteurs. Il s'attache aussi à en développer des "animations," sortes d'applications de leur pensée aux productions littéraires ou artistiques (comme Apocalypse Now de Francis Ford Coppola) ou activité (la danse Cajun à la Nouvelle-Orléans) qu'elle interpelle selon lui. ${ }^{1}$

1 Charles J. Stivale, The Two-Fold Thought of Deleuze and Guattari: Intersections and Animations (New York: Guilford, 1998). 
croisements Deleuze / Robbe-Grillet sont bilatéraux: Deleuze fait appel aux textes et aux films du nouveau romancier, et Robbe-Grillet a déclaré lui-même qu'il "évoque souvent l'image que Gilles Deleuze donne de la structure dans Logique $d u$ Sens [où] une structure serait comme deux séries parallèles, qui ne sont pas de même nature - disons qu'il y aurait une série de concepts et une série d'objets. ${ }^{2} \mathrm{Si}$ la série d'objets est indubitablement indicatrice de l'oeuvre de Robbe-Grillet, celle faite de concepts définit la pensée critique de Gilles Deleuze. Il l'a dit et répété: "la philosophie est l'art de former, d'inventer, de fabriquer des concepts." 3

Certains commentateurs ont ainsi déjà signalé, en clair ou par allusions, les correspondances entre ces deux auteurs. Dès l'origine de la critique américaine sur le Nouveau Roman, Ben Stoltzfus indiquait $L$ 'Anti-Oedipe à ses lecteurs pour les aider à comprendre la particularité du 'désir' robbe-grillétien. ${ }^{4}$ Dans son étude de la fonction de l'érotisme dans les écrits et les films de Robbe-Grillet, c'est le texte de Deleuze sur

2 Voir Roger-Michel Allemand, Alain Robbe-Grillet (Paris: Seuil, 1997) 194, et Gilles Deleuze, Différence et Répetition (Paris: Presses Universitaires de France, 1968) 376; Cinéma 2- L'image-Temps (Paris: Minuit, 1985) Chapitres 3, 5,6 et 9; L'Abécédaire de Gilles Deleuze, dir. Pierre-André Boutang, prod. Sodaperaga, eds. Montparnasse, 1989. Entretiens filmés avec Claire Parnet, diffusés sur la chaine Arte entre 1994 et 1995, où le philosophe explique dans "L comme Littérature" qu'il lui aura fallu cinq ans pour comprendre le genre d'innovation que faisait Robbe-Grillet. Dans "O comme Opéra" Deleuze approuve Robbe-Grillet quand celui-ci dit que Balzac était un génie mais qu'il n'y a aucun intérêt à écrire des romans à la Balzac aujourd'hui.

${ }^{3}$ Gilles Deleuze et Felix Guattari, Qu'est-ce que la Philosophie? (Paris: Minuit, 1991) 8.

4 Ben Stoltzfus, Alain Robbe-Grillet: The Body of the Text (Cranbury: Associated University Press, 1985) 74. 
aider à comprendre la particularité du 'désir' robbe-grillétien. ${ }^{4}$ Dans son étude de la fonction de l'érotisme dans les écrits et les films de Robbe-Grillet, c'est le texte de Deleuze sur Sacher-Masoch que Raylene L. Ramsay citait. ${ }^{5}$ Tout récemment, Roger-Michel Allemand se référait nominalement à Logique du Sens et Cinéma 2: L'Image-Temps et il renvoyait, indirectement, à Deleuze quand il inscrivait RobbeGrillet "dans la lignée d'un Bergson.",

Mais les croisements ne s'arrêtent pas là. Il me semble, de plus, que Robbe-Grillet met en forme, si ce n'est en "scène," les fondements de la pensée deleuzienne: "Ils ont à présent un air attentif et sage. Ils veulent voir la suite. Ils sont au théâtre, et moi sur la scène, en train de jouer une pièce inconnue, qu'un étranger a écrite pour moi..." (ARG: Djinn, 41) Bien au-delà des élans mytho-politico-poétiques de Capitalisme et Schizophrénie, en-deça de la rigueur de Différence et Répétition ou Logique du Sens, et en parallèle à la critique cinématographique de Deleuze, les objets littéraires de Robbe-Grillet composent, à son insu, une série d'incorporations anorganiques des concepts-clés qui reviennent, éternellement, dans toute l'oeuvre de Deleuze.

Ce projet d'analyse veut donc lire le texte, à mon avis, robbe-grillétien par excellence: Djinn: Un trou rouge entre les pavés disjoints, à partir de concepts deleuzien majeurs que je

4 Ben Stoltzfus, Alain Robbe-Grillet: The Body of the Text (Cranbury: Associated University Press, 1985) 74.

5 Raylene L. Ramsay, Alain Robbe-Grillet and Modernity (Gainesville: University Press of Florida, 1992) 159. Voir Gilles Deleuze, Présentation de Sacher Masoch (Paris: Minuit, 1967).

6 Roger-Michel Allemand, Alain Robbe-Grillet (Paris: Seuil, 1997) 68. Voir aussi Gilles Deleuze, Cinémal: L'image-Mouvement (Paris: Minuit, 1983) et Le bergsonisme (Paris: Presses Universitaires de France, 1966). 
mettrai en séries. ${ }^{7}$ En filigrane aux errances dans Djinn, je propose de considérer, tout d'abord, l'aggrégat des concepts deleuziens de "segmentarité," et d' "agencements." Ceux-ci entrainent l'étude comparée qui suivra des notions de "territoire," "lignes de fuite" et "devenir." Pour retracer l'antidéveloppement du personnage métamorphe de Simon Lecoeur, j'invoquerai, ensuite, la "schizoanalyse" de Deleuze et Guattari et leur conceptualisation d'un "Corps sans Organes." Devenir et corps étant intimement liés à une conception particulière du Temps et dans ce "roman," et pour Deleuze, c'est enfin dans la perspective d'un 'Nouveau Baroque' que j'envisagerai Djinn, à partir du concept de

${ }^{7}$ Bien que Djinn n'ait pas, pour l'instant, fait l'objet de travaux critiques spécifiques amples, je le considère tout particulièrement robbegrillétien. A paraître, cependant "Djinn d'Alain Robbe-Grillet: Eros au coeur de l'andro-gyne fragmenté" de Christian Milat dans Le Nouveau Roman en questions, Roger-Michel Allemand, ed. Probablement parce que le texte est, à l'origine, un manuel scolaire destiné aux étudiants de Français à New York University, il ne bénéficie pas du même statut critique que les autres romans de Robbe-Grillet. Il en est cependant la quintessence puisqu'il inscrit le travail de la langue et dans sa forme et dans son objet. De plus, ayant été écrit pour le marché académique américain, il ne comporte aucun des développements érotiques et/ou pornographiques et/ou sadiques qui sont un des fameux leit-motives de Robbe-Grillet. S'il est indéniable que ces derniers sont constitutifs de sa thématique, et responsables en partie de l'impact de son oeuvre, il me semble cependant qu'ils participent d'un Robbe-Grillet autre que celui du Nouveau-Romancomme-projet-pour-une-révolution-à-Paris. Rien de moins nouveau, effectivement, que ces descriptions lancinantes de tortures et autres charcutages du corps féminin. Il ne s'agit pas ici de condamner cet aspect de l'oeuvre, mais simplement de le poser en additif à sa qualité intrinsèque. Pour une étude extensive du traitement du corps féminin, voir Raylene $L$. Ramsay, "Myths of Woman or The Sadist and the Siren" ch. 4 in Alain Robbe-Grillet and Modernity (Gainesville: University Press of Florida, 1992). Djinn, étant en quelque sorte débarrassé d'un tel poids, permet un décodage plus neutre de ce que Barthes appela si joliment "l'écriture: ce compromis entre une liberté et un souvenir." Degré 0 de l'écriture (Paris: Seuil, 1953) 16. 
"pli." Il s'agit, en somme, de voir en Djinn, une animation au sens stivalien du terme - deleuzienne de l'appareil robbegrillétien. $^{9}$

Bien que ce ne soit qu'en 1976 que le mot "topologie" apparait en tant que tel dans l'univers de Robbe-Grillet, tous les critiques s'accordent à le dire construit, depuis son origine, par et entre d'innombrables lignes, traits, segments et autres fils conducteurs qui ne mènent jamais nulle part mais ramènent toujours à son oeuvre. ${ }^{10}$ Selon l'écrivain, le roman moderne serait un microcosme du monde lui-même, lieu aliénant où le lecteur en fiction, comme de fait, ne cesse de se perdre (ARG: $P N R, 116$ ). Stoltzfus, qui insère sa lecture dans un cadre structuraliste, considère que la topologie labyrinthique des lieux et des textes de Robbe-Grillet participe de l'élaboration de différents niveaux qui "transcendent souvent la dimension réflexive du langage." empreinte de théorie féministe, voient en les pérégrinations et les déambulations difficiles des personnages masculins une éternelle tentative de retour à la matrice ou une constante aspiration vers un précipice vaginal édenté. ${ }^{12}$ Quant à

${ }^{8}$ Voir la bibliographie de Robbe-Grillet, Deleuze, Deleuze et Guattari en fin de document. Pour faciliter la lecture, les titres sont mentionnés en abrégé directement dans le texte.

${ }^{9}$ Par deleuzien, je n'entends pas effacer l'apport immense et crucial de Guattari à la pensée de Deleuze, mais simplement alléger les références.

${ }^{10}$ Il s'agit, bien sûr, de Topologie d'une cité fantôme.

${ }^{11}$ Ben Stoltzfus, Alain Robbe-Grillet: The Body of the Text (Cranbury: Associated University Press, 1985) 18.

12 "The Return is itself threatened by the perilous rising tides, the encroachment of the sea, the addying pools that suck men down in the tentacles of their strange depths." Raylene L. Ramsay, Alain Robbe-Grillet and Modernity (Gainesville: University Press of Florida, 1992) 65. 
Allemand, il considère que les ruptures narratives traduisent les "béances" qui "trouent" une existence où "le souvenir fait défaut." 13

Il me semble que de telles exégèses, toutes cohérentes qu'elles soient, tendent à enfermer les textes de Robbe-Grillet dans des grilles interprétatives trop contraignantes. Voyez le structuralisme: c'est un système de points et de positions qui opère par grandes coupures dites signifiantes, au lieu de procéder par poussées et craquements, et qui colmate les lignes de fuite, au lieu de les suivre, de les tracer, de les prolonger dans un champ social. (GD: Dial, 48)

Dans la mesure où tous les textes de Robbe-Grillet se dés-articulent autour des mêmes rappels des textes précédents, fantasmes et hachures diégétiques, ces éléments structurels perdent leur valeur constitutive et transcendantale. En clair, à partir du troisième "marin péri en mer" et de la deuxième allusion à un monstre marin, il devient difficile de continuer à voir, en toute cette eau, le symbole d'un quelconque refoulement (ARG: LMR, 94, 98). Robbe-Grillet n'a laissé récemment plus aucun doute sur la valeur pseudo référentielle ou révélatrice de son travail linguistique:

Le langage "articulé", j'insiste à nouveau là-dessus, est structuré comme notre conscience claire, ce qui revient à dire: selon les lois du sens. Il se trouve ainsi, par voie d'immédiate conséquence, incapable de rendre compte d'un monde extérieur qui précisément n'est pas nous, et des spectres qui s'agitent à l'intérieur de notre corps. Mais, en même temps, il me faut bien l'utiliser, ce matériau-là, le langage, si inadapté soit-il, puisque c'est

${ }^{13}$ Roger-Michel Allemand, Alain Robbe-Grillet (Paris: Seuil, 1997) 22. 
cette conscience claire - et rien d'autre - qui se plaint du non-sens et du manque. (ARG: $L M R, 41$ )

L'inconcient aura été un langage, mais le langage, lui, parle la conscience. Après cinquante ans d'écriture, les découvertes psychanalytiques sont devenues des clichés, et, continuer, par exemple, à lire 'mère' au lieu de "mer" ancre le texte dans un passé et une extériorité qu'il entend précisément reconfigurer.

De même, s'il est vrai que la double réflexivité des écrits de Robbe-Grillet - retour sur l'écriture et échos de ses autres écrits - a été probablement le facteur d'innovation le plus puissant dans les Années Soixante, à nouveau, après quarante ans du même procédé, cette réflexivité est désormais monument historique plus que drapeau révolutionnaire. Et pourtant, Robbe-Grillet continue à fasciner car il ne cesse pas d'être fascinant.

La question n'est aujourd'hui plus pourquoi mais: comment? En ce qui concerne Djinn, en tout cas, aucun des modèles - linguistique/structuraliste, féministe/études culturelles ou psychologique - n'y répond de façon complètement satisfaisante. Précisément parce qu'il n'y a aucune transcendance et que les fantasmes sont réduits à de simples fétiches (mannequin, chandeliers, tissu de robe et trou de boue rouillée, entre autres). Quant à la "fragilité mentale de Boris/Simon/Jean," dont parle Allemand, celle-là même qui se traduirait par ses amnésies et le décuplement de sa personnalité, c'est un critère psychologique surprenant à propos d'un texte qui cherche à s'écarter de tout psychologisme. Comment, dès lors, cartographier Djinn non pas dans sa 'modernité' mais dans sa constante contemporanéité, son être-là ici et maintenant? 
Peut-être en commençant par voir en ses "pavés disjoints," ceux dont l'inégalité [des deux pavés] avait prolongé les images desséchées et minces que j'avais de Venise dans tous les sens et toutes les dimensions, de toutes les sensations que j'y avais éprouvées, raccordant la place à l'église, le canal à l'embarcadère, et à tout ce que les yeux voient le monde de désirs qui n'est vu que de l'esprit. (P: ARTP III, 876)

$\mathrm{Au}$ lieu de considérer les pavés, et, par extension, les segments narratifs de Djinn, comme étant séparés par un trou, disjoints, je propose de les voir plutôt rassemblés -"joints"autour de cet espace, toujours encore à combler, -“dis"perséau travers de temps et lieux différents et différés. Dans son roman Swann, comme le remarque Genette, Proust parlait de "la solidarité qu'ont entre elles les différentes parties d'un souvenir et que notre mémoire maintient équilibrées dans un assemblage où il ne nous est pas permis de rien distraire ni refuser." ${ }^{\prime 14}$ Les pertes de conscience ou de mémoire, les points de suspension qui scandent le récit dans Djinn, les nombreuses allusions à la sensation d'être "hors du temps" (ARG: Djinn, 30), à un "temps arrêté" (ARG: Djinn, 31), pour "combien de temps? (ARG: Djinn, 33) ne contribuent pas à éparpiller la mémoire ou fragmenter la conscience, mais, au contraire, à les rapprocher différemment: non comme masse, solide et univoque mais comme "assemblage" faits des "linéaments qui traversent aussi bien des groupes que des individus." (D\&G: $M P, 249)$

Il n'y a, en fait, ni transcendance ni individualité dans Djinn. Si quelque chose y est clair, c'est que Boris Koershimen, ingénieur électronicien à Kiev, et/ou Robin Körsimos, professeur à l'école de Passy, dit Simon Lecoeur,

14 Gérard Genette, Figures III "Métonymie chez Proust" (Paris: Seuil, 1972) 41-63. 
appelé Yann - épelé Ján par ses élèves n'est ni même ni autre que le Boris du Régicide, Boris Godounov dans le Miroir qui revient, Boris "l'homme qui ment" et/ou Coeur Simon dans Angélique ou l'enchantement. Pour emprunter la terminologie deleuzienne, le personnage de Robbe-Grillet est un "agencement," à savoir un "système sémiotique/expression régime de signes" doublé d'un "système pragmatique/contenu - actions et passions." (D\&G: $M P, 629)$ Comme le suggèrent Deleuze et Guattari, "entre les deux, entre le contenu et l'expression, un nouveau rapport s'établit [...]: les énoncés ou les expressions expriment des transformations incorporelles qui s'attribuent comme tels (propriétés) aux corps et aux contenus." (D\&G: $M P, 629)$

J'aurai ainsi tendance à proposer que la logique du sens qui structure l'oeuvre de Robbe-Grillet n'est pas son éclatement ni sa fragmentation mais, au contraire, son unité, son regroupement autour d'un espace "entre" et d'un temps 'autre' qui fonctionnent comme catégories à priori de son entendement. ${ }^{15}$ De même que les personnages de Beckett, que convoquent Deleuze et Robbe-Grillet, "se font un territoire de leur poubelle ou de leur banc," le personnage central mais excentrique de Djinn ne cesse de se constituer, de façon autonome malgré tout, un territoire entre des repères qui lui sont imposés. ${ }^{16}$ Si les bribes d'actions se passent à l'intérieur

15 La terminologie kantienne n'est pas employée ici par inadvertance. Malgré la tendance à considérer le Kantisme comme antagoniste à la pensée de Deleuze, et en dépit des propos mêmes de ce dernier, si Deleuze rejette toute considération "a-priori," sa conception du temps et de l'espace n'est pas aussi radicalement opposée à celle de Kant qu'on pourrait le croire. Mais une telle démonstration serait l'objet d'un tout autre article -- à venir. Voir Gilles Deleuze, La philosophie critique de Kant (Paris: Presses Universitaires de France, 1963)

${ }^{16}$ Mille Plateaux, 629. "Pour peu, emporté par mon élan, je tomberais aussi sur lui, et nous roulerions ensemble dans le noir, l'un par dessus l'autre, comme des personnages de Samuel Beckett." Djinn, 103. 
d'un lieu précis (le hangar, la maison, le café, le taxi, la salle de réunion), la passion de Simon (curiosité dévorante ou amour pour Jean-Djinn) croît dans les intervalles de son 'voyage:' lorsqu'il interrompt celui-ci pour sauver l'enfant chapitre 2-, discuter avec la petite Marie -chapitre 3-, raconter une "histoire d'amour et de science-fiction" -chapitre 4-, tricher pendant la réunion -chapitre 5-, questionner Jean chapitre 6-, ou aimer Marie jeune fille -chapitre 7- ${ }^{17} \mathrm{C}$ 'est le déplacement entre (autant que la lutte des) sexes qui est "moteur de l'histoire," mais celui-ci se fait sur place. A partir d'une dynamique de la stase, les intermittences réorganisent et l'histoire dans Djinn et son historique dans le corpus entier. On passe insensiblement non seulement d'un point à un autre, mais d'un espace-temps à un autre, ou simplement d'un livre à un autre. En ce sens, le texte est bien contruit en "strates: phénomènes d'épaississement sur le Corps de la terre: accumulations, coagulations, sédimentations, plissements." (D\&G: MP , 627) Et ses différents plateaux mènent sur la piste de Jean-Djinn ou s'empilent dans l'oeuvre globale de RobbeGrillet.

Mais c'est aussi le passage de livre-racine à livreradicelle que le texte informe en le formalisant. Du premier, Deleuze et Guattari écrivent qu'il est "image du monde [...] belle intériorité organique signifiante et subjective." Sa loi serait "celle de la réflexion, le Un qui devient deux." (D\&G: $M P$, 11) A l'opposé, le livre-radicelle aurait vu sa racine principale avorter, "se détruire vers son extrémité," mais sur elle se serait greffée "une multiplicité immédiate et quelconque de racines secondaires qui prennent un grand développement." L'unité d'un tel livre serait "passée ou à venir, possible." (D\&G: MP,12) Et ce livre, "image du monde

${ }^{17}$ Par souci de simplicité, j'appellerai le personnage central de Djinn Simon bien qu'il ne s'agisse d'omettre aucune de ses variantes. 
chaos, chaosmos radicelle" serait "d'autant plus total que fragmenté." (D\&G: MP, 13) L'unité de Djinn est souterraine (soutextuelle), elle réside dans les trous, plongeant vers les textes antérieurs, mais aussi dans les seuils que le roman entrouvre sur ceux à venir. Djinn, comme texte articulation, tend vers, autant qu'il attend, des pavés à sa périphérie.

De même, ce qui définit Simon est une intensité, une capacité à (re)devenir telle ou telle figure, à prendre tel ou tel visage (professeur, marin, espion-terroriste, jeune femme américaine), mais à ne jamais pouvoir être enfermé dans un territoire précis:

Voilà que, dans la rupture, non seulement la matière du passé s'est volatilisée, mais la forme de ce qui s'est passé, d'un quelque chose d'imperceptible qui s'est passé dans une matière volatile, n'existe plus. On est devenu soi-même imperceptible et clandestin dans un voyage immobile. Plus rien ne peut se passer, ni s'être passé. Plus personne ne peut rien pour moi, ni contre moi. Mes territoires sont hors de prise, et pas parce qu'ils sont imaginaires, au contraire: parce que je suis en train de les tracer. [...] Je n'ai plus aucun secret, à force d'avoir perdu le visage, forme et matière. Je ne suis plus qu'une ligne. Je suis devenu capable d'aimer, non pas d'un amour universel abstrait, mais celui que je vais choisir en aveugle, un double, qui n'a pas plus de moi que moi. (D\&G: $M P, 244)$

Ces réflexions étaient faites, par Deleuze et Guattari, à propos de Henri James, mais elles s'appliquent remarquablement à la fois au personnage et à l'auteur de Djinn quand il l'écrit. Non seulement Simon est devenu "imperceptible," dilué dans la mythique centième page de son 
récit qui n'a pas été retrouvée, mais Robbe-Grillet semble enfin "rencontrer le vrai double à l'autre bout de la ligne (Corinthe?) [...] devenir comme tout le monde, mais justement ce n'est un devenir que pour celui qui sait n'être personne, n'être plus personne [qui sait se peindre] gris sur gris." (D\&G: MP, 241)

Peu surprenant, dès lors, que l'oeuvre du romancier prenne un tournant radical après Djinn, moment qu'Allemand appelle, à juste titre, "la renaissance autoscripturale" des Miroir qui revient, Angélique ou l'enchantement et Les derniers jours de Corinthe. Lequel se produit, chronologiquement, après un hiatus de sept ans. (ARG: $L M R, 7$ ). Comme le ferait la conclusion d'une "shizo-analyse" dégageant des "lignes qui peuvent être aussi bien celles d'une vie, d'une oeuvre littéraire ou d'art, d'une société, d'après tel système de coordonnées retenu," Djinn signale et signe la fin du processus de déterritorialisation de ses personnages et le début de la reterritorialisation de Robbe-Grillet.

C'est un argument quelque peu, mais non moins correct, dans la mesure où le texte se devait, en lui-même déjà, d'être une tentative d'occupation simultanée de plusieurs territoires:

Et l'un des charmes dont se pare à mes yeux la vie de professeur d'université, que je mène de temps à autre en Amérique (à New York ou sur les campus perdus des immenses Etats aux noms légendaires), c'est que j'y redeviens aussitôt étudiant. Elèves studieux (les miens sont "gradués"), discussions théoriques avec les autres enseignants, douillette atmosphère de ghetto culturel, d'exterritorialité (hors nation, hors temps)... (ARG: $L M R$, 203) 
Visiblement, le texte-manuel scolaire s'insère mais par la bande, entre parenthèses en quelque sorte, à l'interieur du marché académique aux Etats-Unis "ensemble molaire aux segments durs, surcodés par l'[es] Etat[s]" s'il en est. (D\&G: $M P$, 249) Plus subtilement, le roman à pour discrète watermark les passations de pouvoir qu'ont connu les départements d'études françaises des campus américains dans les années 80. Que ce soient par les filles qui "ne sont plus comme autrefois [...] violent les adolescents sans défense, portent des pantalons", les pizzas qui tuent, le - mauvais - café américain ou à travers la critique du machinisme, Le Rendezvous s'inscrit dans une lutte de partage d'un territoire (prof. hommes vs. profs. femmes, études littéraires vs. culturelles, enseignants français vs. américains, francais vs. espagnol etc.) en plein rétrécissement. ${ }^{18}$ Mais il le fait depuis la position privilégiée de son auteur, professeur célèbre, étranger et, donc, toujours dans un territoire à part.

Djinn propose ainsi une ligne de fuite, ligne qui "ne consiste pas à fuir le monde mais plutôt à le faire fuir" par "coupures" asignifiantes (D\&G: MP, 249). Au-delà des questions de différe/ance-s, et de l'interrogation légitime "qu'est-ce qui s'est passé?" le texte fond disjonction et conjonction, fiction et réalité, auteur et personnage, adulte et enfant, homme et femme. Tout individu y est multiple, un groupe, tout groupe éclaté: même la sur-présence de l'écrivain-narrateur-personnage est définie, a contrario, par

${ }^{18} \underline{\text { Le Rendez-vous, }}$ publié par Holt, Rinehart et Winston en 1981 est le texte original de Djinn: Un trou rouge entre les pavés disjoints. Il a été écrit par Robbe-Grillet et conçu par Yvone Lenard pour servir de manuel d'apprentissage progressif des difficultés de la grammaire française. Il y est fait référence en page 10 de Djinn, et est présenté comme le texte intégral du manuscrit de Simon Lecoeur. Lequel manuscrit ne comportait donc ni le prologue ni l'épiloque qui encadrent la version française. 
son absence. Ce qui reste de toute cette histoire, sa trace, c'est le sous-titre (seul et unique dans l'oeuvre de Robbe-Grillet). Et c'est pourquoi le trou rouge entre les pavés disjoints réunit, plutôt qu'il ne les sépare, les morceaux des territoires en présence en les rendant, via la tekhnê (art et métier) du nouveau roman, compossibles.

Aussi, la répétition dans Djinn se fait le "mouvement créateur d'un espace et d'un temps dymaniques" que distingue Deleuze. La répétitivité robbe-grillétienne est "intensive," elle "comprend la différence," se comprend elle-même dans l'hétérogénéité d'une 'apprésentation." (GD: $D \& R, 36) \mathrm{La}$ répétition confère son authenticité à un langage plus que jamais trompeur mais elle circonscrit les écueils de la signification. Elle contribue à aplanir la narration, en enveloppant les résurgences d'action qui divisent le récit dans un amalgame de lieux-topoi d'une étrange familiarité.

Cependant, on assiste à un véritable recommencement dans Djinn. Très français malgré tout, le corps insaisissable de Jean/Djinn est celui d'une tabula rasa -- clean slate ou clean stone. ${ }^{19}$ Comme le Jean Santeuil (cent oeil et non sans oeil) de la Recherche, Djinn/Jean a retrouvé sa "Marie Kossichef," dans ce qui sert d'hôtel de 'passe-âge' aux enfants et la Marie de Simon y est parfaitement lisse, un pur esprit. Proust écrivait:

19 “Les Anglais, les Américains n'ont pas la même manière de recommencer que les français. Le recommencement français, c'est la table rase, la recherche d'une première certitude, comme d'un point d'origine toujours le point ferme. L'autre manière de recommencer, au contraire, c'est reprendre la ligne interrompue, ajouter un segment à la ligne brisée, la faire passer entre deux rochers, dans un étroit défilé, ou par dessus le vide, là où elle s'était arrétée." Gilles Deleuze et Claire Parnet. Dialogues (Paris: Flammarion, 1996) 50. 
Quelquefois en passant devant l'hôtel il se rappelait les jours de pluie où il emmenait sa bonne, en pélerinage. Mais il se les rappelait sans la mélancolie qu'il pensait alors devoir goûter un jour dans le sentiment de ne plus l'aimer. Car cette mélancolie, ce qui la projetait ainsi d'avance sur son indifférence à venir, c'était son amour. Et cet amour n'était plus. (P: $J S, 674)$

Le "parfait zigzag" des positions temporelles dont parle Genette à propos de ce passage évoque celui des escaliers de New York ou ceux qui traversent la mémoire de l'enfant Jean quand il se "rappelle avec une précision extraordinaire, ce qui n'est pas encore arrivé." (ARG: $P R N Y$, 81 - Djinn, 112) Que le petit garçon convoque ici et maintenant "le moi actuel" de Simon alors que celui-ci est ailleurs, ou qu'il rappelle Marie morte depuis trois ans, il fonctionne non seulement comme guide spatial, mais comme intercesseur temporel qui unifie tout, en aspirant protagonistes, siècles, actions et oeuvres dans la béance pleine de sa mémoire. (ARG: Djinn, 112-13)

Pour qui se rappelle Deleuze, ces lacérations extra- et diégétiques évoquent la dernière entrée du philosophe dans son Abécédaire: " $Z$ comme Zigzag." Chez Deleuze, le $Z$ est le mouvement de la mouche, le mouvement "élémentaire," celui du big Bang à l'origine des choses. ${ }^{20} \mathrm{Le} \mathrm{Z}$ mettrait "en rapport des singularités disparates ou des potentiels" et entre les deux potentiels réunis par sa figure, c'est un éclair qui apparaitrait,

20 Voir les entretiens télévisés entre Claire Parnet et Gilles Deleuze ou consulter leur résumé en anglais établi par Charles J. Stivale à l'adresse suivante:

Cs.html

http://www.langlab.wayne.edu/Romance/FreD_G/FRED\%26GAB 
et rendrait visible "l'événement," à savoir "ce qui est arrivé à la chose, soit qu'elle le subisse, soit qu'elle le fasse." (GD: Pli, 55) ${ }^{21}$

Les zigzags à la fois hyper- et paramnésiques qui strient le sommeil de l'enfant et provoquent les pertes de conscience de Simon matérialisent le nouvel événement Robbe-Grillet, le produit d'une conscience enfin détachée de la transcendance d'un inconscient figuratif, et fondamentalement freudien. En clair, le trou bée sur la seule immanence de l'oeuvre elle-même. Dans Djinn, "l'inconscient ne désigne plus le pan caché du plan d'organisation transcendant, mais le processus du plan de consistance immanent," c'est-à-dire que ni le pavé-début du texte (ce qui s'est passé avant six heures et demi) ni le pavé-fin du texte, la centième page, comme manque, n'importent. Rien n'est à trou-ver, tout est vu entre, "l'intéressant c'est le milieu" (D\&P: Dial, 50): la matière, la boue des personnages, des situations, des références, des événements, en résumé des 'milieux' robbe-grillétiens eux-mêmes.

Si le trou est rouge, ce n'est plus parce qu'il préfigure le drame, meurtre ou autre violence à laquelle le lecteur habitué s'attend, mais parce que le rouge est "solution radicale à l'irréductible antagonisme entre le noir et le blanc." (ARG: $P R N Y, 38)$ Le rouge ici trace une ligne, même si elle est brisée, qui joint les deux contraires. Il ne s'agit pas de tenter un mélange - impossible - ce serait le seul gris des pavés disjoints, mais d'embrasser l'écart. Le rouge se fait ligne de fuite hors, précisément, de la dialectique. Paradoxalement, il permet également de sortir du voyeurisme. La couleur démythifiée redouble l'aveuglement volontaire de Simon parce qu'elle n'explique jamais la mort dans ce texte:

${ }^{21}$ Il s'agit ici de la "chose littéraire." 
"Comme celui-ci [le récit de Simon Lecoeur] l'avait signalé, la flaque de sang était artificielle. Le médecin légiste a tout de suite constaté que le corps ne comportait aucune blessure, ni aucun traumatisme externe." (ARG: Djinn, 144)

A l'opposé de celui de la victime de "La chambre secrète" par exemple, les corps dans Djinn se font lisses et donc impénétrables. (ARG: Inst, 107) Tracés entre les lignes des rapports policiers mais non striés par elles ni sous celles des lames habituelles, les corps disparaissent mais pour mieux paraitre unifiés en-deça de toutes coupures textuelles et, par la-même, échapper aux lacérations sexuelles et au démembrement psychanalytique.

Le corps dans Djinn ne se s'étale plus. Il ne se montre pas mais resurgit en des points qui jalonnent un "plan de consistance." Il est "corps sans organes" parce qu'habité d'intensités: celles qui incorporent chacun des personnages de Djinn dans la répétition et leur différence, celles traçant des lignes en provenance de et vers l'avant- et l'après- Djinn, celles qui génèrent les événements par jeu de variations et assemblage de variantes. ${ }^{22}$ Depuis son origine, l'oeuvre de Robbe-Grillet tue et efface le corps de l'être individuel pour composer un continuum dont la corpo-réalité apparait en trompe-l'oeil, d'un segment de l'oeuvre sur l'autre: Il prit la coupure de journal et, [...] relut le texte attentivement, une fois de plus, d'un bout à l'autre. On n'y disait en réalité pas grand'chose. La longueur ne dépassait pas celle d'un fait divers de seconde importance. Encore, une bonne moitié ne faisait-elle que retracer les circonstances oiseuses de la découverte du corps; comme toute la fin était consacrée à des commentaires sur l'orientation que les gendarmes donnaient aux recherches, il restait fort peu de lignes pour la description

22 Voir la conclusion de Mille Plateaux, 632-34. 
du corps lui-même [...] Il fallait réinventer la scène d'un bout à l'autre à partir de deux ou trois détails élementaires... (ARG: $L V, 75-76$ )

Chaque corps est pris dans un devenir-variante de soi et de son altérité, et ne consiste que de créations "qui augmente[nt] le nombre des connexions" avec les scènes et les textes qui l'entourent. (D\&G: $M P, 634$ ) Il ne s'agit pas de métamorphoses, mais de traits pointillés qui dessinent la carte de l'univers robbe-grillétien, "carte d'intensité qui distribue les affects dont la liaison, la valence constituent chaque fois l'image du corps, image toujours remaniable ou transformable dans la mesure des constellations affectives qui la déterminent." (GD: Cri-Cli, 84) Chaque voyage, chaque trajet - dans l'île du Régicide, suivant le boulevard circulaire des Gommes, le long des rochers du Voyeur, sur les pistes de $L a$ Jalousie...- est un tracé à la fois chaotique et cosmogonique.

Contrairement à ce qu'il semble, et en dépit de la pertinence de l'analyse d'Allemand, ces tra-jets/cés ne mènent pas plus à des "impasses" qu'à des "issues." 23 Car, finalement, il ne s'agit jamais de 'sortie' chez Robbe-Grillet mais toujours d'entrée, dans une nouvelle "disposition" des personnages (ARG: $L J, 107$ ) ou dissonance des textes. Ce qui est mis en forme est une insertion de la langue dans la langue, du sens dans le non-sens, de la collectivité dans "l'énonciation individuée." C'est pourquoi on peut voir en Robbe-Grillet un nouvel exemple de la "littérature mineure" que définissent Deleuze et Guattari.

Celle-ci aurait les principes suivants:

- la littérature mineure n'est pas celle d'une langue mineure, plutôt celle d'une minorité (ici, les nouveaux

${ }^{23}$ Roger-Michel Allemand, Alain Robbe-Grillet (Paris: Seuil, 1997) 85 . 
romanciers) fait dans une langue majeure (la tradition romanesque française),

"l'espace exigu" de la littérature mineure "fait que chaque affaire individuelle est immédiatement branchée sur la politique. L'affaire individuelle devient donc d'autant plus nécessaire, indispensable, grossie au microscope, qu'une tout autre histoire s'agite en elle:" (D\&G: $K, 30)$

[...] les centaines de feuillets manuscrits que comporte déjà mon rapport, surchargés de ratures mais rangés en bonne ordonnance (notes préliminaires marquées, en haut à droite, d'un $\mathrm{O}$ suivi du numéro d'ordre et quelquefois aussi d'une date, brouillon $\mathrm{A}$, brouillon $\mathrm{B}$, brouillon $\mathrm{C}$, numérotés à leur tour, bien entendu, feuilles de rajouts ou de récritures concernant des passages exagérément corrigés, grossis, devenus indéchiffrables pour moi-même [...] (ARG: $A E, 126-27$ ),

- parce que "les conditions ne sont pas données d'une énonciation individuée, qui serait celle de tel ou tel "maître," [...] ce que l'écrivain tout seul dit constitue déjà une action commune, et ce qu'il dit ou fait est nécessairement politique [...], c'est la littérature qui se trouve chargée positivement de ce rôle et de cette fonction d'énonciation collective, et même révolutionnaire: c'est la littérature qui produit une solidarité active, malgré le scepticisme; et si l'écrivain est en marge ou à l'écart de sa communauté fragile, cette situation le met d'autant plus en mesure d'exprimer une autre communauté potentielle, de forger les moyens d'une autre conscience et d'une autre sensibilité" (D\&G: $K, 32$ ),

- finalement, dans la littérature mineure, "un roman ne devient un roman même s'il n'est pas achevé, même et surtout s'il est interminable, que si les indices machiniques 
s'organisent en un véritable agencement consistant par luimême:" (D\&G: $K, 69)$

Les feuilles inférieures, dont les angles dépassent de tous les côtés de façon très irrégulière, sont hachurées par les lignes serrées d'une écriture soigneuse. Celle du dessus, la seule à être visible toute entière, n'est encore écrite qu'à moitié; elle se termine, au milieu d'une ligne, par une phrase interrompue, sans aucun signe de ponctuation après le dernier mot. (ARG: Inst, "Scène," 56)

C'est, paradoxalement, parce qu'elles suivent ces ''lois,' que l'oeuvre de Kafka, dont parlent Deleuze et Guattari, et celle de Robbe-Grillet sont "révolutionnaires," comme le dit Stoltzfus. ${ }^{24} \mathrm{Ou}$, tout au moins, elles participent d'un devenir-révolutionnaire, même si et surtout parce qu'elles échappent et à l'Histoire et à l'histoire. ${ }^{25}$ Comme pour celle de Kafka, on peut dire que l'écriture de RobbeGrillet a la "double fonction de transcrire en agencements et de démonter les agencements." (D\&G: $K, 86$ ) Il y a, chez Robbe-Grillet, un 'esprit systématique' à l'oeuvre si puissant qu'il combat celui de ou du système signifiant jusqu'à lui prévalent: l'indétermination et l'infinitude du sens, comme

${ }^{24}$ Ben Stoltzfus, Alain Robbe-Grillet: The Body of the Text (Cranbury: Associated University Press, 1985) 59.

25 Deleuze clarifie la différence, cruciale pour sa pensée, entre "devenir des gens" et "avenir de l'histoire" dans son entretien télévisé avec Claire Parnet à la lettre "G comme Gauche." Il dénonce "la confusion du devenir et de l'histoire" et montre que ce n'est pas "l'avenir révolutionnaire" mais "comment et pourquoi les gens deviennent révolutionnaires" qui importe. Son raisonnement est fondé sur le postulat que le fait - évident pour lui - que les révolutions n'ont pas d'avenir n'implique pas qu'il n'y ait pas de devenir-révolutionnaire possible. 
signification, sensation et direction, sont les facteurs primordiaux de la minorisation de la langue.

A propos de Dans le Labyrinthe, Allemand parlait de "circularité du parcours" des personnages. ${ }^{26}$ Celle-ci est, en effet, indéniable dans ce texte ou les autres. Mais elle provient moins d'un enroulement du parcours autour de lui-même que d'un repli de chaque personnage, objet, lieux sur eux-mêmes où il est difficile, sinon impossible, de retrouver début et fin. Le système de Robbe-Grillet consiste à renvoyer "l'ensemble de la matière à une courbure qui n'est plus déterminable du dehors. La courbe suivie par un corps quelconque sous l'action de l'extérieur renvoie à une unité «supérieure» interne et individuante, à l'autre étage, et qui contient «la loi de la courbure» la loi des plis ou des changements de direction." (GD: Pli, 18)

Sous l'effet des effets volontairement appuyés de retard, d'échos, de rappels, de renvois - quasi encyclopédiques dans Djinn - à une tradition elle-même de réitération, le textepavé absorbe ses propres trous et acquiert la plasticité d'une pâte (boue) à modeler et à moduler. Le cadre du marin péri en mer, par exemple, n'est pas une fenêtre ouvrant sur le reste de l'oeuvre en tant qu'extérieur du texte mais la manifestation ostensible d'un de ses nombreux plis internes. Suivant la définition qu'en donnait Heinrich Wölflin, la vision que ce cadre permet est celle d'une image baroque: "vision picturale opposée à la vision linéaire de la Renaissance, profondeur et surface, unité et multiplicité, clarté et obscurité."27 Si l'on a pu assimiler les techniques narratives du nouveau roman à

${ }^{26}$ Roger-Michel Allemand, Alain Robbe-Grillet (Paris: Seuil, 1997) 85.

${ }^{27}$ Benito Pelegrin, "Visages, Virages, Rivages du Baroque, Rêves et Dérives" in Jean-Marie Benoist, ed. Colloque de Cerisy: Figures du Baroque (Paris: Presses Universitaires de France, 1983) 19. 
celles, picturales, du cubisme et proposer que son monde soit "privé de profondeur," dans la mesure ou l'art -littéraire et visuel - ne voudrait plus désormais copier la nature, peut-être faudrait-il aussi les comparer à celles de la représentation baroque. Allemand parlait des "volumes dépliés" du cubisme. ${ }^{28}$ Stolztfus faisait des "miroirs, tableaux et fenêtres" les marqueurs d'une intertextualité visant à contrecarrer toute entreprise mimétique, semblable aux efforts de Picasso. ${ }^{29}$ En comparant ce type de répétitivité à la dépiction cubiste, on présente le texte robbe-grillétien comme étalé, mis à plat, étiré entre deux références ou trois rappels dans un processus de continuité. Allemand suggère que les "tableaux, images, photographies, affiches, statues et poupées participent d'une mise en abyme où se dissout la référentialité. ${ }^{30}$

Je propose d'y voir le contraire, une accentuation, valorisation et célébration de la référentialité comme force active au sens Nietzschéen du terme, affirmant l'être du texte en tant que création, et de la littérature en tant que vie:

Voilà donc tout ce qu'il reste de quelqu'un, au bout de si peu de temps, et de moi-même aussi bientôt, sans aucun doute: des pièces dépareillées, des morceaux de gestes figés et d'objets sans suite, des questions dans le vide, des instantanés qu'on énumère en désordre sans parvenir à les mettre véritablement (logiquement) bout à bout. C'est ça, la mort... Construire un récit, ce serait alors - de façon

${ }^{28}$ Roger-Michel Allemand, Alain Robbe-Grillet (Paris: Seuil, 1997) 14.

${ }^{29}$ Voir Ben Stoltzfus, Alain Robbe-Grillet: The Body of the Text (Cranbury: Associated University Press, 1985) 136, 151.

${ }^{30}$ Roger-Michel Allemand, Alain Robbe-Grillet (Paris: Seuil, 1997) 21 . 
plus ou moins consciente - prétendre lutter contre elle. Tout le système romanesque du siècle dernier, avec son pesant appareil de continuité, de chronologie linéaire, de causalité, de non-contradiction, c'était en effet comme une ultime tentative pour oublier l'état désintégré où nous a laissés Dieu en se retirant de notre âme, et pour sauver au moins les apparences en remplaçant l'incompréhensible éclatement des noyaux épars, des trous noirs et des impasses par une constellation rassurante, claire, univoque, et tissée à mailles si serrées qu'on n'y devinerait plus la mort qui hurle entre les points, au milieu des fils cassés renoués à la hâte. (ARG: $L M R, 27)$

Loin de tomber dans l'abîme de l'abyme, ou de dissoudre la référentialité dans la répétition, il me semble que l'oeuvre de Robbe-Grillet se meut dans ce que Deleuze oppose à la continuité, à savoir la contiguïté. Au lieu de se dérouler le long de singularités, de points repérables par leur récurrence, textes et oeuvre se froissent dans le plissé d'un véritable lexique événementiel et objectif. Un tel catalogue est comparable à celui des objets que re-présentent les tableaux baroques, dont plusieurs ne sont pas sans rappeler ceux qui jalonnent l'univers de Robbe-Grillet: chandelles éclairant le visage d'une jeune femme (ARG: Djinn, 35, 110); manuscrits et feuillets (ARG: Inst. "Scène," 59); lourds rideaux ou vêtements rouges (ARG: Djinn, 115); crucifix (ARG: Djinn, 190); sols - maçonniques? - carrelés (ARG: Djinn, 74); lits de mort (ARG: Djinn, 110); tissus blancs et vaporeux (ARG: Djinn, 36, 108); objets à la signification codée; plans de villes aux perspectives rectilignes et croisées. ${ }^{31}$

31 Pour quelques exemples d'images robbe-grillétiennes et baroques concernant: 
Dans Djinn, par exemple, le cadre du marin apparait sept fois (ARG: Djinn, 38, 46, 92, 116, 117, 118, 119). Tout comme l'objet baroque, "son propos n'est pas de restaurer la valeur absolue et universelle de la forme mais d'affirmer ouvertement la valeur autonome et intrinsèque de l'image., ${ }^{., 2}$ Le marin, par son aspect changeant et accidentel, ne renvoie à rien d'autre qu'à une autre image similaire à la fois interne et externe au texte. Il est image, mais celle du persona de la reférentialité et non d'un personnage, rappel de l'existence

Chandelles éclairant le visage d'une jeune femme: voir George de La Tour, La Madeleine à la veilleuse et Saint Sébastien soigné par Irène, Musée du Louvre, Paris.

Manuscrits et feuillets: voir Ribera, Saint Jerôme et l'ange, Musée de L'Hermitage, St Petersbourg; et sa série des philosophes grecs: Diogène, Collection privée; Anaxagore, Collection privée, Platon, Los Angeles County Museum of Art, Protagoras, Wadsworth Atheneum, Hartforf; Cratyle, The National Museum of Western Art, Tokyo; Aristote, Indianapolis Museum of Art.

Lourds rideaux ou vêtements rouges: voir Caravage, $\underline{\text { La mort de la }}$ Vierge, Musée du Louvre, Paris; Velázquez, Vénus au miroir, National Gallery, Londres.

Crucifix: voir Guerchin, La Crucifixion, Chiesa del Rosario, Cento.

Sols carrelés: voir Vermeer, L'atelier - Allégorie de la peinture, Kunsthistorisches Museum, Vienne; La lettre, Rijksmuseum, Amsterdam.

Lits de mort: voir Zurbaran, Les Funérailles de Saint Bonaventure, Musée du Louvre, Paris.

Tissus blancs et vaporeux: voir Jean-Honoré Fragonard, Fontaine d'amour, Collection Wallace, Londres.

Objets à la signification codée: voir Jacques-Louis David, La Vision de Saint Jérôme, Cathédrale de Québec.

Plans de villes aux perspectives rectilignes et croisées: voir Christopher Wren, Vue du Dôme et plan de la cathédrale St Paul, Londres. 1989) 10.

${ }^{32}$ Giulio Carlo Argan, L'Age baroque (Genève: Eds. Albert Skira, 
d'un monde possible, non parallèle mais plié dans la réalité littéraire. L'assemblage de ces marins entraine à jouer le "jeu d'analogies, d'associations, de combinaisons, de contaminations, ne se pliant plus qu'à des exigences occasionnelles" qui caractérise la forme baroque. ${ }^{33}$ De même ce genre d'agencement préside à la mémoire involontaire de Proust "raccordant la place à l'église, le canal à l'embarcadère, et à tout ce que les yeux voient le monde de désirs qui n'est vu que de l'esprit." (P: ARTP III, 876) L'image conserve une structure, un cadre au propre comme au figuré, mais elle est décuplée, trouvant son sens dans les perspectives de l'oeuvre toute entière, dans les plis et les trous de textes contigüs.

Robbe-Grillet donne ainsi un parfait exemple de la multiplicité dont parle Deleuze dans son étude des "plateaux" comme dans son analyse de Leibniz et du Baroque. Pour le philosophe, "le multiple est inséparable des plis qu'il fait quand il est enveloppé et des déplis qu'il fait quand il est développé." (GD: Pli, 33). Et c'est bien ce que le romancier force son lecteur à faire: envelopper et développer sans cesse les pièces de son patchwork. Qu'on le veuille ou non, comment lire un de ces romans sans le placer dans "la triade du pli:" c'est à dire "l'expliquer, l'impliquer, le compliquer" dans et par ses autres Mêmes, dès l'instant où on en a lu ne serait-ce qu'un? (GD: Pli, 33)

Conformément aux théories de Deleuze et Guattari,

le multiple, il faut le faire, non pas en ajoutant toujours une dimension supérieure, mais au contraire le plus simplement, à force de sobriété, au niveau des dimensions dont on dispose, toujours $\mathrm{n}-1$ (c'est 1989) 11.

${ }^{33}$ Giulio Carlo Argan, L'Age baroque (Genève: Eds. Albert Skira, 
seulement ainsi que l'un fait partie du multiple, en étant toujours soustrait). Soustraire l'unique de la multiplicité à constituer; écrire à $\mathrm{n}-1$. (D\&G: $M P, 13)$

Dans Djinn, comme dans ses textes contigüs, le lecteur doit toujours chercher le 1 ailleurs, plié à la connexion avec 'l'extérieur' de ce texte-ci, c'est-à-dire 'l'intérieur' du reste de cette oeuvre-là. Le trou de Djinn est $\mathrm{n}$ moins le 1 des trous, creux, bosses et ornières sur l'ile du Voyeur (ARG: $L V, 155-$ 56), et-et-ou de celui qui coupe la piste dans La Jalousie (ARG: $L J, 166-67)$. Sa couleur est $\mathrm{n}$ moins celle génératrice du Projet pour une Révolution à New York, roman où l'on trouve aussi le reste de l'unité du personnage central Laura, simple collaboratrice dans Djinn (ARG: PRNY, 28). Les chandelles et le Dr. Morgan sont $\mathrm{n}$ moins les 1 de celles avec lesquelles il torture Christine dans Souvenirs $d u$ Triangle d'Or. La gare du Nord trouve son 1 manquant par assonnance avec l'Edouard Nordman de La Maison de Rendez-Vous. Le mannequin est réunifié dans celui qui ouvre les "Trois visions réfléchies" des Instantanés. Quant aux "marins péris en mer qui viennent tirer les vivants par leurs pieds dans leurs lits" (ARG: $L M R, 20$ ), ils sont pur matière baroque qui "a tendance à sortir du cadre" (GD: Pli, 166). Simplement, parce que "le cadre n'est pas suffisant pour contenir la masse qui déborde et passe par-dessus." (selon Heinrich Wölflin dans GD: Pli, 167).

Et quand le cadre figure la mort, écriture et image se doivent d'être devenir-vie. Ainsi, de même que le Baroque "se définit par le pli qui va à l'infini," l'oeuvre de Robbe-Grillet n'est pas retour, ni renvoi mais pliage sur elle-même, origami d'emboitements d'illusion de présence, d'hallucinations de consistance, de mirages fluides mais réels. Pour en conclure à travers Deleuze: 
C'est l'objet même qui est élargi, suivant tout un réseau de relations naturelles, c'est lui qui déborde son cadre pour entrer dans un cycle ou une série, et c'est le concept qui se trouve de plus en plus resserré, rendu intérieur, enveloppé dans une instance qu'on peut dire "personnelle" à la limite. (GD: Pli, 171)

\section{A quoi Robbe-Grillet a bien répondu:}

Je n'ai jamais parlé d'autre chose que de moi. Comme c'était de l'intérieur, on en s'en est guère aperçu. (ARG:

$L M R, 10$.)

L'auteur, jusqu'à l'après Djinn, aura été devenuimperceptible.

Un homme est une particule insérée dans des ensembles instables et enchevêtrés. Ces ensembles composent avec la vie personnelle à laquelle ils apportent des possibilités multiples [..] A partir de la connaissance, l'existence d'une personne n'est isolée de celle de l'ensemble que d'un point de vue étroit et négligeable. Seule l'instabilité des liaisons (ce fait banal: quelque intime que soit un lien, la séparation est aisée, se multiplie et peut se prolonger) permet l'illusion de l'être isolé, replié sur lui-même et possédant le pouvoir d'exister sans échange.

Georges Bataille - L'expérience intérieure. 